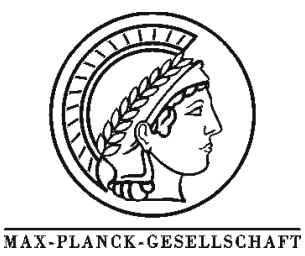

\title{
Interaction of diamond with water: an in situ XANES investigation
}

\author{
P. Reinke, ${ }^{a}{ }^{*}$ A. Knop-Gericke ${ }^{\mathrm{b}}$, M. Hävecker ${ }^{\mathrm{b}}$, Th. Schedel-Niedrig ${ }^{\mathrm{b}}$ \\ ${ }^{\text {a } I n s i t u t ~ f u ̈ r ~ P h y s i k, ~ U n i v e r s i t a ̈ t ~ B a s e l, ~ K l i n g e l b e r g s t r . ~ 82, ~ C H-4056 ~ B a s e l ~}$ \\ ${ }^{b}$ Department of Inorganic Chemistry, Fritz-Haber-Institute of the MPG, Faradayweg 4-6, 14195 Berlin, Germany \\ * Corresponding author: e-mail reinke@ubaclu.unibas.ch, phone +41 612673 7145, fax +41 612673784
}

Received 24 September 1999; accepted 18 November 1999

\begin{abstract}
The interaction of water and oxygen with a polycrystalline diamond film was investigated using XANES ( $\mathrm{X}$-ray absorption near edge structure) spectroscopy. A novel reactor design allowed to access pressure ranges up to $4.0 \mathrm{mbar}$ and simultaneous recording of the gas phase and sample spectra at the $\mathrm{C}-\mathrm{K}$ and $\mathrm{O}-\mathrm{K}$ edges, respectively. The diamond surface is inert with respect to the interaction with water in the investigated te $\mathrm{m}-$ perature below $500^{\circ} \mathrm{C}$. A defect ( $\mathrm{sp}^{2}$ carbon) rich surface layer, is created through ion irradiation $\left(\mathrm{Ar}^{+}, 2 \mathrm{keV}\right)$, and the ratio of the $* / *$ resonance was used as a measure of the extent of damage. A partial removal of the damage is observed in the first few minutes of water exposure, but a steady-state concentration of defects remains even after prolonged exposure periods. The resultant steady -state damage is nearly independent of the water pressure. The reaction of the damaged surface with water leads also to the atta chment of oxidic groups, mostly in the form of carbonyl groups, to the surface. The carbonyl groups seem to be located mostly at the surface and result directly from the reaction with dangling bonds or $\mathrm{sp}^{2}$-carbon defects with water. The irradiation with oxygen ions leads likewise to an overall reduction in damage, the chemcial etching dominates the creation of new defects through ion impact.
\end{abstract}

Keywords: Chemisorption; Diamond; Ion bombardment; Near edge extended X-ray absorption fine structure (NEXAFS); Oxidation; Polycrystalline surfaces; Water

\section{Introduction}

Understanding the interaction between the diamond surface and various reactands, such as e.g. water or oxygen, is of considerable interest for the application of these films as sensors or in electrochemical processes. The inertness of the diamond surface ${ }^{1}$ has been exploited to develop devices which function in environments hostile to other materials. The transformation of the diamond surface through exposure to hydrogen and other adsorbants 2,3 has been investigated in recent years, mainly in conjunction with the aim to understand the diamond growth process, but the interaction with water is only poorly understood ${ }^{4-6}$. Oxygen, however, can not be attached easily to diamond surfaces ${ }^{7}$ and the appearance of oxygen containing surface terminating groups is only observed after heating in an oxygen atmosphere ${ }^{8}$, or exposing ion irradiated diamond surfaces to atomic oxy- gen 9 . These experiments indicate that acitve sites at the diamond surface or a highly reactive oxygen species are required to enhance the surface reactivity. The ion irradiation of diamond leads to the formation of a defect rich surface layer, which is structurally similar to an amorphous carbon film ${ }^{10,11}$ (and references therein) but does not exhibit a long range graphite-like ordering. The damage layer contains a large concentration of $\mathrm{sp}^{2}$ hybridized carbon atoms and dangling bonds which can subsequently serve as reaction centers.

We describe in the following paragraphs an experiment aimed to study the reactions of diamond and defect-rich diamond surfaces with water and its influence on surface composition and defect density. 


\section{Experimental}

The in-situ observation of the reactions is possible by performing X-ray absorption measurements of the near edge structure (XANES) in a tank reactor designed to operate in the pressure range between $1 \sum 10^{-8}$ to 20 mbar. A detailed description of the reactor is given by Knop-Gericke et al. in a recent publication ${ }^{12}$. For the investigation of the reactivity of the diamond surface a polycrystalline, boron-doped diamond film with random orientation of the crystallites was used as substrate. The XANES measurements were performed at the HE-TGM-1 (Berliner Elektronenspeicherringgesellschaft für Synchrotronstrahlung, BESSY I) beamline ${ }^{13}$ using the first-order light of the monochromator. The sample spectra presented here are recorded with the total electron yield (TEY) collection plate located in front of the substrate and the sample current induced by relaxation processes in the sample at both normal and, in some cases, grazing incidence (an angle of $55^{\circ}$ with respect to the surface normal was chosen). Gas phase spectra are obtained from the collection grid, which is located at a larger distance from the sample and close to the polyimide window, separating the processing chamber from the beamline. The carbon K-edge spectra obtained at the collection plate and the sample current are divided by the collection grid signal, thus correcting for the window transmission and variation in beam current with time and energy at the same time. The carbon K-edge jump is subsequently normalized to unit height at the maximum of the * resonance peak, which is the most intense peak in all spectra. For the correction of the window transmission and beam profile of the oxygen $\mathrm{K}$ edge a different method was applied in order to avoid complications which arise from reactions of the grid and collector with the water atmosphere. Surface oxides at the collection grid will contribute to the spectra as an additive component rather than a multiplicative one and therefore introduce artificial features in the spectra if they are used in the correction procedure in the way described for the carbon K-edge. All spectra (collector, collection grid and sample) are divided by the equivalent spectrum (collector, collection grid and sample, respectively) recorded in vacuum $\left(10^{-7}\right.$ mbar) with a pristine sample prior to each water experiment; this leads to a correction of all spectra with respect to the window transmission and beam profile. The sample signal at the collection plate can be separated from the gas phase signal by subtraction of the corrected collection grid signal (dominated by the gas phase contribution) after normalization of the spectra to unit height. It has been ascertained that this correction method is also applicable for the C-K edge and delivers equivalent spectra as the method described in the previous paragraph.

\section{Results and discussion}

In a first set of experiments the hydrogen-plasma cleaned diamond sample was exposed to water vapour in the pressure range between $1 \sum 10^{-4}$ and 2 mbar for extended periods of time. The carbon K-edge spectra of the sample recorded at different times during the exposure at the TEY collection plate are shown in figure 1 . The corresponding processing sequence of the samples is summarized in table 1 . The energy of the $\sigma^{*}$ resonance is adjusted at $290 \mathrm{eV}$, the value which is given in the literature for diamond $10,14,15$.

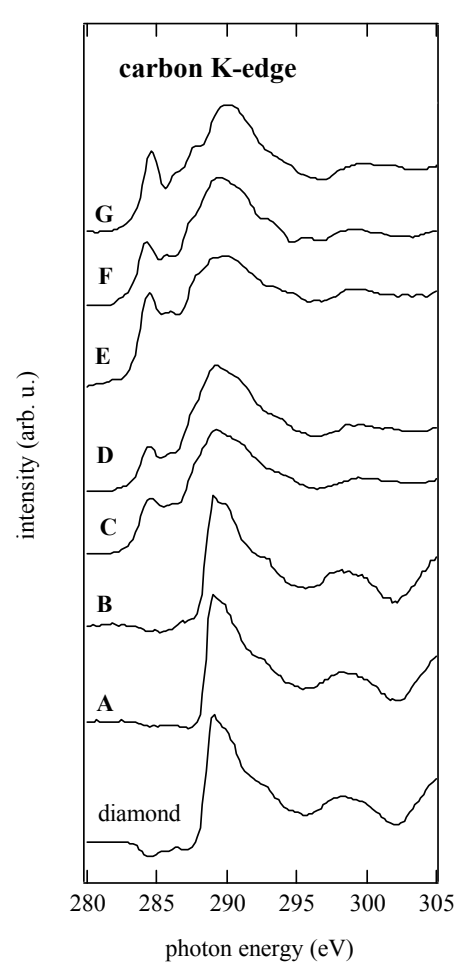

Fig. 1: Carbon K-edge spectra recorded at different stages of the modification of diamond films through the irradiation with ions $\left(\mathrm{Ar}^{+}, \mathrm{O}_{2}^{+}\right)$and the exposure to water at different pressures. The processing conditions and assignment of the spectra is summarized in table 1 . The spectra are normalized to unit height at the position of the $\sigma^{*}$ resonance.

The relative peak positions in the spectrum of the original diamond surface are in agreement with published data $10,14,15$ and a prominent K-edge absorption feature, the $\sigma^{*}$ resonance, located at $290 \mathrm{eV}$, a shoulder at $292 \mathrm{eV}$ and the second gap in the unoccuppied density of states at 302.5 $\mathrm{eV}$ can be identified. Although the relative peak positions agree excellently with the literature data, the intensity of the $\sigma^{*}$ resonance peak as compared to the peak, which appears just as a shoulder around $292 \mathrm{eV}$, differs from some of the literature data. This disagreement might be attributable to the random orientation of the crystallites, which is in contrast to most other studies usually performed on single crystals or textured films. Upon exposure to water no significant changes in the spectra are observed indicating the inertness of the diamond surface against the reaction with $\mathrm{H}_{2} \mathrm{O}$ at low substrate temperatures. Annealing of the sample in a water atmosphere (at 0.2 and $2.0 \mathrm{mbar}$ for $10 \mathrm{~min}$, and at $4.0 \mathrm{mbar}$ for $40 \mathrm{~min}$ ) at $480^{\circ} \mathrm{C}$ does likewise not lead to detectable changes in the carbon $\mathrm{K}$-edge structure (see figure 1, spectrum A and B). The oxygen K-edge spectrum indictates the presence of oxidic structures, but the small signal intensity prohibits a more specific assignment. The reactivity of the undamaged diamond surface towards water is apparently very low, and even elevated substrate temperatures lead to only minute changes in the surface composition. 
A second set of experiments was performed by creating a damaged diamond surface through the irradiation with $\mathrm{Ar}^{+}$ ions $(2 \mathrm{keV})$ prior to exposure to $\mathrm{H}_{2} \mathrm{O}$. The damage accumulates in the surface region and leads to the formation of a highly defective diamond layer which resembles structurally

Table 1: Summary of processing conditions for the diamond films included in figure 1 (carbon K-edge) and figure 3 (oxygen K-edge). The spectra were measured in vacuum unless otherwise indicated.

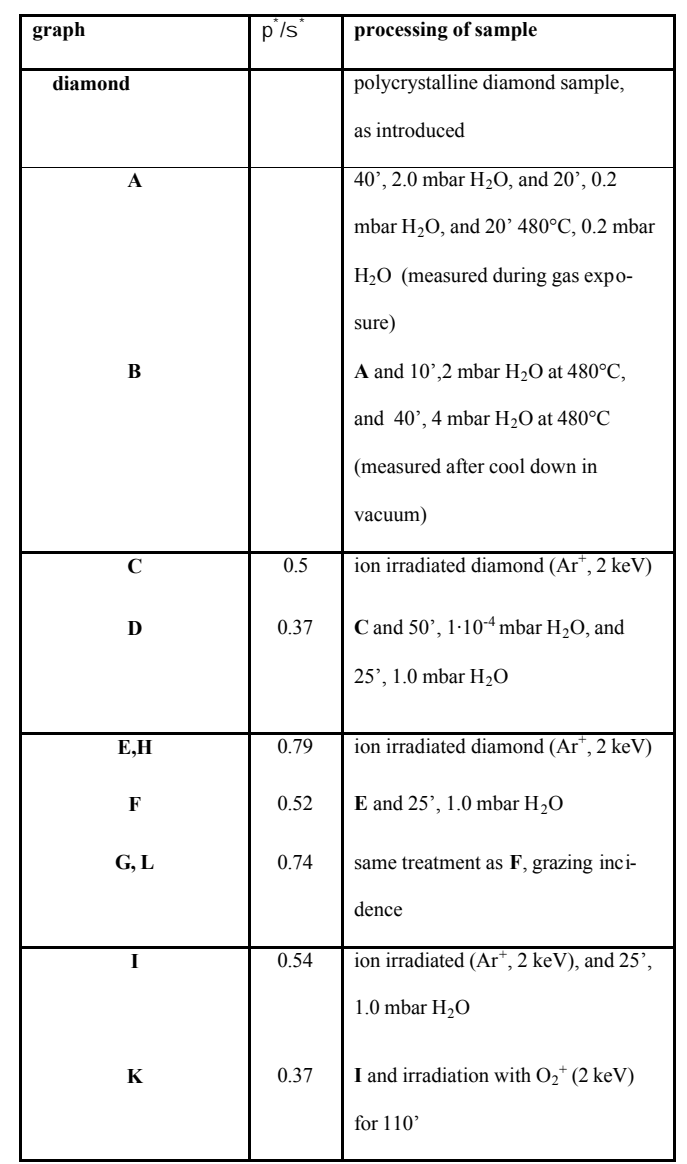

$\mathrm{sp}^{2}$-rich amorphous carbon ${ }^{10,11}$. This structural modification is also readily apparent in the carbon K-edge XANES spectra ((see fig 1, spectra $\mathrm{C}$ through $\mathrm{G})$ : an additional peak, the $\pi^{*}$ resonance, appears at $285 \mathrm{eV}$ and the $\sigma^{*}$ resonance peak is broadened. However, the structures which appear on the high binding energy side of the $\sigma^{*}$ resonance are still preserved, although somewhat smeared out, and signify the persistence of a long range order ${ }^{10,16}$. A small feature appears around $286.3 \mathrm{eV}$ and is a constant contribution to all spectra (even the diamond spectra) and the only feature in the spectra which might be an artefact related to a structure in the absorption spectrum of the polyimide window, separating the beam vacuum chamber and the reaction chamber. A reliable separation of this feature from any possible contribution to the $\mathrm{C}$ K-edge from carbonyl groups can not be achieved. An alternative assignment of this feature could also be the presence of $\mathrm{C}-\mathrm{H}$ groups at the surface, which would also be in agreement with the angular dependence of this peak's intensity (fig. 1, spectra F and G).
Although the diamond features are still apparent in the region above $300 \mathrm{eV}$ after ion irradation, they appear smeared out, which is directly connected to the relaxation of kconservation and gradual transition to the unstructured density of final states characteristic for an amorphous material, and the emergence of states in the gap. The intensity of the $\pi^{*}$ resonance with respect to the $\sigma^{*}$ resonance is a direct measure of the defect density and can be used to monitor the changes in the film damage $10,16,17$. Exposure of these damaged films to $\mathrm{H}_{2} \mathrm{O}$ leads indeed to a decrease in the intensity of the $\pi^{*}$ resonance. This observation is illustrated in figure 1 , showing a selection of spectra before and after $\mathrm{H}_{2} \mathrm{O}$ exposure, and in figure 2 , where the intensity ratio of the $\pi^{*}$ to $\sigma^{*}$ resonance is shown as a function of exposure time to water. Data for two films with a different initial damage level, which were exposed to water at varying pressures are included. In both cases a rapid decrease in $\pi^{*} / \sigma^{*}$ ratio takes place in the first minutes of water exposure at $10^{-4} \mathrm{mbar}$, and a constant level of damage is then retained for longer exposure times. Increase in the water pressure to 1.0 mbar leads to a slight decrease in the $\pi^{*} / \sigma^{*}$ ratio, but no further reduction is observed after subsequent annealing at $450 \infty \mathrm{C}$. This general behaviour has been observed repeatedly at different $\mathrm{H}_{2} \mathrm{O}$ pressures, however, a complete removal of the damaged surface layer can not be achieved even at pressures exceeding 2.0 mbar. The series corresponding to the spectra $\mathrm{E}$ to $\mathrm{G}$ in fig. 1 will be discussed in the next paragraph, in conjunction with the presence of surface oxides.

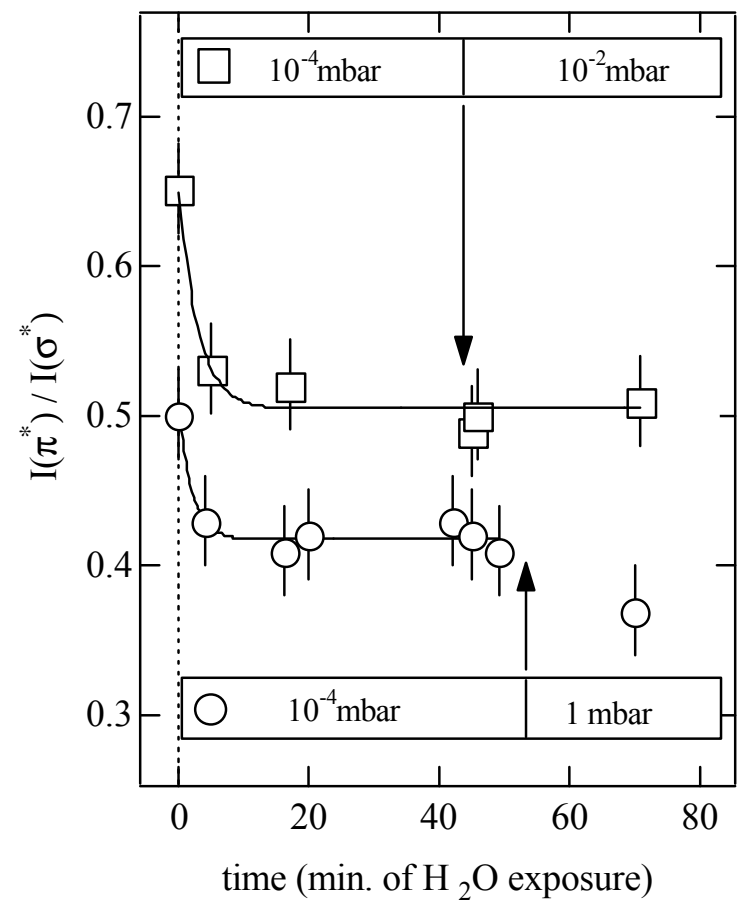

Fig. 2: Modification of the intensity ratio $\pi^{*} / \sigma^{*}$ peak during water exposure at different pressures. Included are the data obtained for two diamond films with different initial damage levels. 
The gas phase spectra (as recorded with the collection grid) are dominated in the O-K edge region by the typical water spectrum ${ }^{14,18}$ (included in figure 2) with a main peak at 535.7 and a shoulder located at lower energies with respect to the most intense peak. The water spectrum was used to calibrate the energy scale in the $\mathrm{O}-\mathrm{K}$ edge region. The con- centration of carbon containing reaction products in the gas phase is apparently very small, and the signal in the C-K edge region remains therefore inconclusive and allows due to its small signal to noise ratio no unequivocal interpretation.

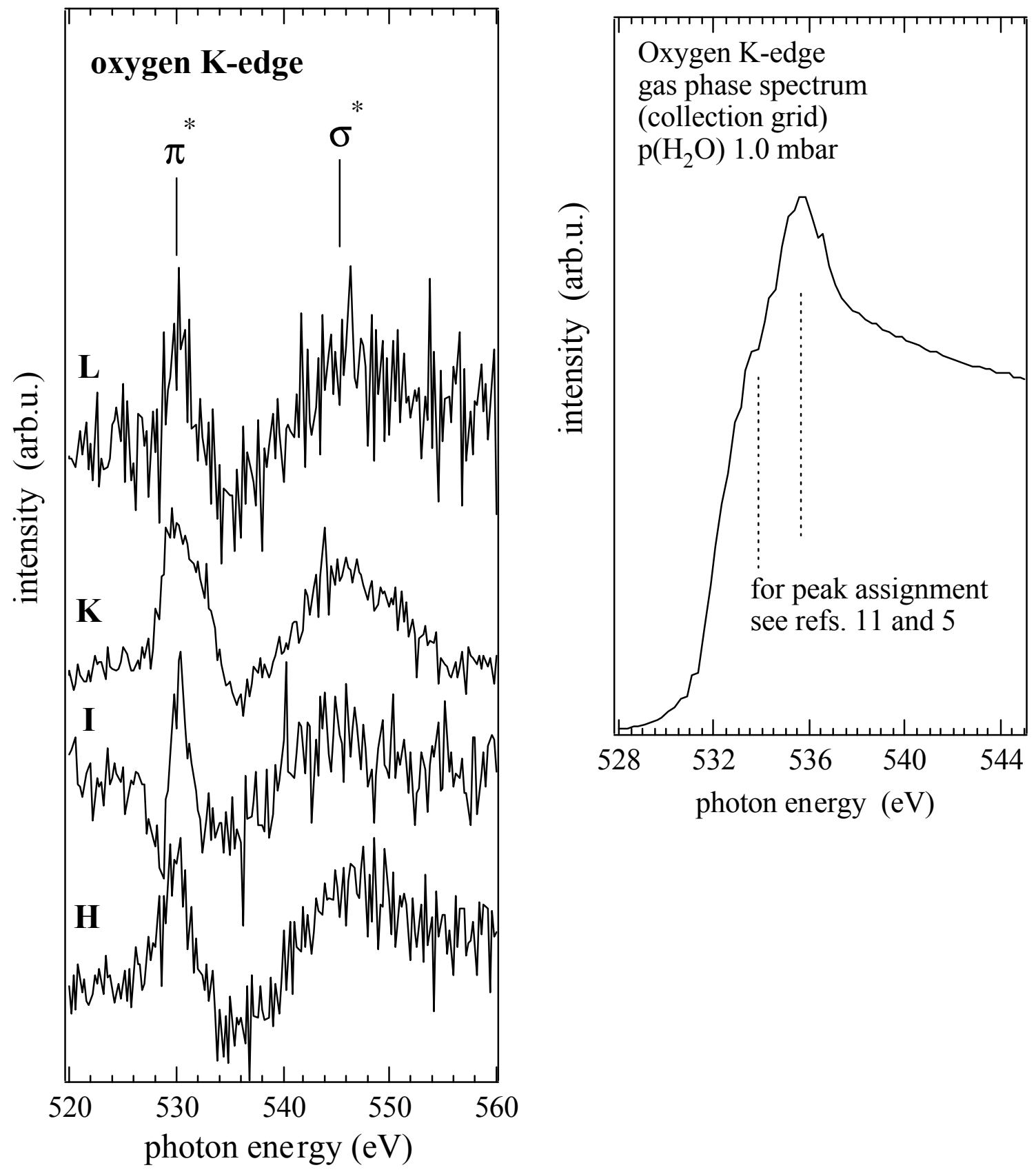

Fig. 3: left hand side: Oxygen K-edge sample spectra recorded at different stages of the modification of diamond films through the irradiation with ions $\left(\mathrm{Ar}^{+}, \mathrm{O}_{2}^{+}\right)$and the exposure to water. The processing conditions and assignment of the spectra is summarized in table 1. For the purpose of illustration the spectra are normalized to unit height at the $\pi^{*}$ resonance. Right hand side: Oxygen K-edge spectrum of the gas phase, measured at the collection grid at a water pressure of 1.0 mbar.

A number of representative sample spectra at the O-K edge recorded at the TEY collection plate, are summarized in figure 3 (spectra $\mathrm{H}$ to $\mathrm{K}$, see also table 1; for the purpose of illustrattion the spectra are normalized to unit height at the $\sigma^{*}$ resonance peak ). They are dominated by one narrow peak located at $530 \mathrm{eV}$, a position characteristic for a $\pi^{*}$ resonance, and a quite broad peak at about $546 \mathrm{eV}$. The peak positions are consistent with a $\mathrm{CO}$ double bond, a carbonyl group $^{14,19}$, which appears to be the dominant oxidic group at the diamond surface, irrespective of the type of treatment. The change in the intensity of the carbonyl signal with processing of the surface is also a measure of the concentration 
of carbonyl groups. For this purpose the intensity of the $\pi^{*}$ resonance peak of the sample current spectrum, after correction for the transmission function, is determined and divided by the beam current. Only spectra measured at pressures below $10^{-6}$ mbar are taken into account to avoid any spurious contributions from the intense gas phase signal.

The lowest intensity of the $\pi^{*}$ resonance is observed directly after irradiation of the diamond sample with $\mathrm{Ar}^{+}$ions $(2$ $\mathrm{keV}$ ), although the formation of some carbonyl or similar groups during sputtering is observed and likely attributable to reactions of the damaged surface with the background gas already at this stage (figure 3, spectrum $\mathrm{H}$ ). Interaction with water or oxygen ions leads to changes in the concentration of the carbonyl group (see following paragraph), but no prominent changes in the shape of the spectra. The presence of other oxidic groups cannot be excluded, and indeed seems likely, but their concentration at the surface is below our detection limit. An exception is spectrum labeled K, obtained after the irradiation with oxygen ions, where a shoulder loctaed around $533 \mathrm{eV}$ is apparent, but the assignment of this feature remains uncertain.
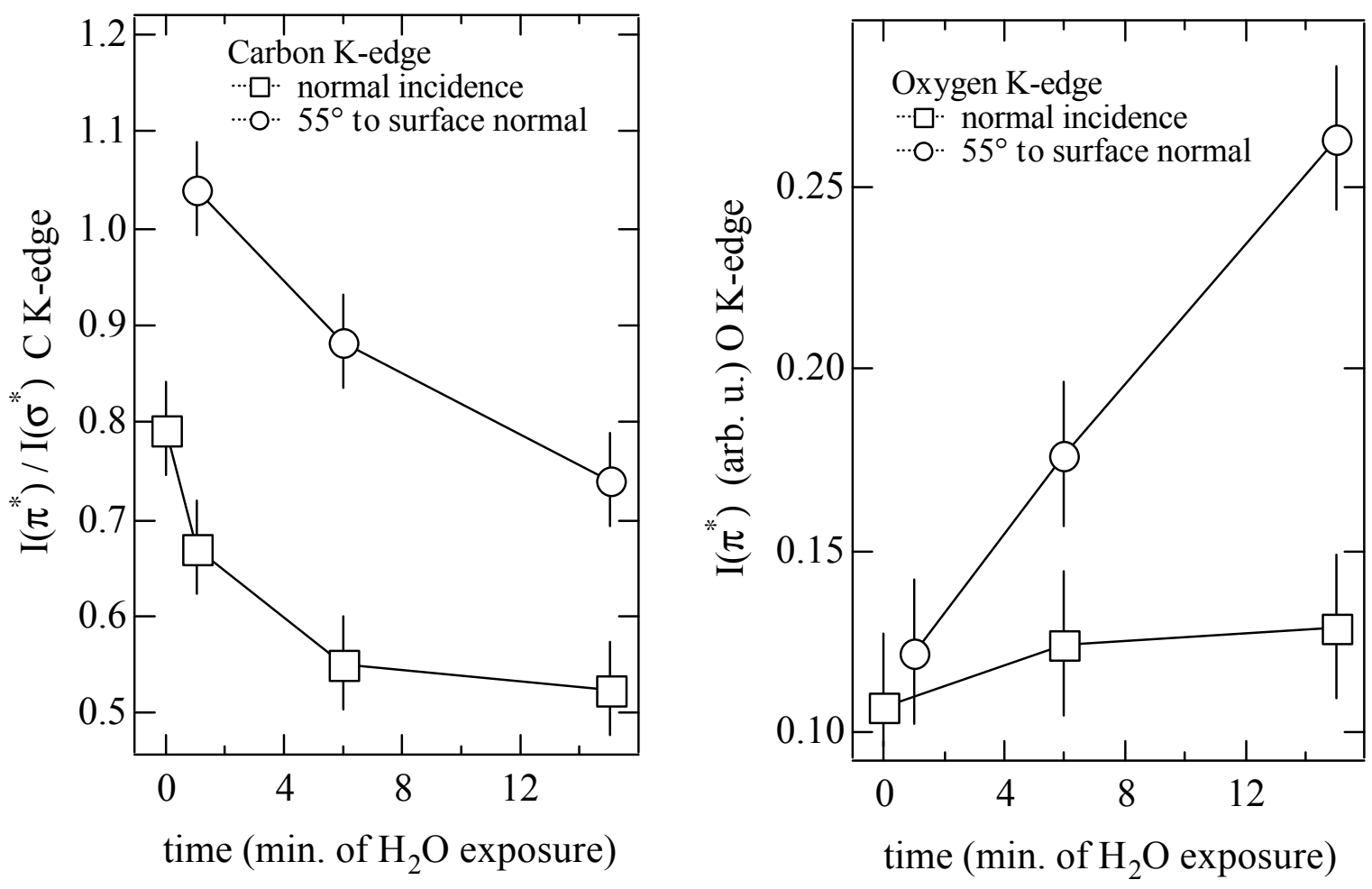

Fig. 4: Comparison of the changes in the $\pi^{*} / \sigma^{*}$ intensity ratio at the C K-edge and the intensity of the $\pi^{*}$ resonance peak at the $\mathrm{O}-\mathrm{K}$ edge as a function of exposure time. The water pressure was $1.0 \mathrm{mbar}$ and the data obtained for measurements performed at normal and grazing incidence of the photon beam are included.

In figure 4 the changes in intensity of the $\pi^{*}$ resonance at the O-Kedge, and in the $\pi * / \sigma^{*}$ ratio at the C-K edge as a function of water exposure time (water pressure of 1 mbar) are illustrated. A selection of spectra recorded during this sequence is included in fig. 1 (C-K edge, spectra $\mathrm{E}$ through $\mathrm{G}$ ) and in fig. 3 (O-K edge, spectrum L). The initial level of damage in this film is quite high as compared to for example sample C (fig.1) due to the larger ion current used during the ion iradiation of the diamond film. The temporal evolution in the reduction in surface defect density as expressed in the decreasing $\pi^{*} / \sigma^{*}$ ratio is similar to the experiment described in the previous paragraph. Although the contribution from the damage layer is larger for grazing incidence, the relative changes in the $\pi^{*} / \sigma^{*}$ ratio are about $30 \%$ for both orientations, owing to the fact that the whole damage layer is accessed for both orientations. Any change in the depth distribution of the damage is masked by the error inherent to the measurement .
At the same time a continuous increase in the intensity of the $\pi^{*}$ resonance at $530 \mathrm{eV}$ and therefore the concentration of carbonyl groups is observed, which is indicative of a reaction of the incoming water molecules with defects. This has been observed repeatedly and the carbonyl group dominates in all cases the O-K edge spectra. The increase in carbonyl concentration is considerably stronger for the measurement at grazing incidence, commensurate with the accumulation of oxygen in the top surface layers.

In figure 5 the effect of oxygen ion irradation on the surface damage $\left(\pi^{*} / \sigma^{*}\right.$ ratio $\mathrm{C}$ K-edge) and the concentration of carbonyl groups at the diamond surface is summarized. Irradiation of the surface with oxygen ions $(2 \mathrm{keV})$ leads to a continuous reduction in the surface damage and a rapid decrease in the concentration of carbonyl groups at the surface. Again the carbonyl group dominates the O-K edge spectra although other oxidic groups such as hydroxide or ether groups might be present in smaller concentrations (fig.3, 
spectra I and K). The quite pronounced reduction in surface damage can be ascribed to a chemical reaction of the incoming oxygen ions preferably with $\mathrm{sp}^{2}$-bonded carbon atoms and a subsequent removal of the volatile reaction products.

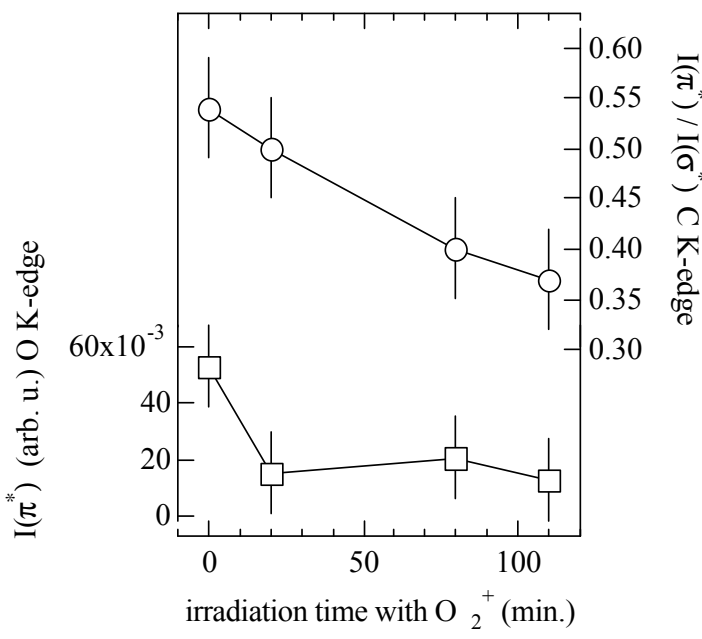

Fig. 5: Intensity ratio $\pi^{*} / \sigma^{*}$ at the $\mathrm{C} \mathrm{K}$-edge, and intensity of the $\pi^{*}$ resonance peak at the $\mathrm{O}-\mathrm{K}$ edge as a function of irradiation time with $\mathrm{O}_{2}^{+}$ions $(2 \mathrm{keV})$.

In this way the oxygen does not contribute to the accumulation of oxidic groups at the surface. The damage created by ion impact is in this case overbalanced by the chemical etching and therefore in marked contrast to the interaction of diamond with $\mathrm{Ar}^{+}$ions, but consistent with the observation of Beerling et al. ${ }^{5}$ on the removal of damaged surface layers with low-energy oxygen ion irradiation

The diamond surface was shown to be indeed rather inert toward the reaction with water, which is in agreement with a study by Struck et al ${ }^{6}$. Defects, on the other hand, serve as reaction centers and the $\mathrm{sp}^{2}$-bonded carbon atoms are readily removed by the interaction with $\mathrm{H}_{2} \mathrm{O}$, but a steady state concentration of defects remains. The defects do apparently not constitute nucleation centers for the etching process, such that numerous active reaction sites remain after the primary interaction of the defect with water. The reaction centers are possibly passivated by the formation of surface oxides, namely the observed carbonyl groups. In the gas phase spectra at the carbon as well as the oxygen K-edge no reaction products can be readily identified, which is attributable to the low efficiency of the reaction and therefore small concentration of reaction products. However, in a thermal desorption study on oxidized diamond powder, Matumoto et al. ${ }^{4,8}$ have shown that the main desorption products are $\mathrm{CO}$ and $\mathrm{CO}_{2}$.

The dominant oxidic group at the diamond surface clearly identifiable after water exposure of defect- rich surfaces, is the carbonyl group with a double bond between the carbon and oxygen atom. The peak positions observed correspond to those known for $\mathrm{HCO}$, and we do not expect a dramatic change in the molecular orbital energies for $\mathrm{CO}$ groups at- tached to a carbon matrix instead of hydrogen due to the small differences in electronegativity between $\mathrm{H}$ and $\mathrm{C}$. The dissociation of water follows therefore a different pathway than on $\mathrm{Si}^{20,21}$, where the formation of hydroxide groups at the surface is observed. The formation of double bonds is energetically highly unfavourable on $\mathrm{Si}$, but apparently the preferred reaction pathway at the diamond surface. The dominance of carbonyl groups at the diamond surface is also observed in the reaction of diamond with oxygen at elevated temperatures ${ }^{8}$, albeit other oxidic groups such as ether or hydroxide groups have also been identified.

The consequences of these findings for practical applications of diamond films are readily apparent. While good quality diamond surfaces are indeed highly inert, defect-rich surfaces which might be produced in the course of the fabrication process of a device, are much more susceptible to degradation. At the same time, the diamond work function is changed quite considerably through the presence of oxygen, and a reaction of the surface leading to the formation of surface oxides can therefore degrade the long term performance of diamond-metal contacts and other electronic devices incoporating diamond films.

\section{Conclusions}

The diamond surface is inert with respect to the interaction with water in the investigated temperature range (below $500^{\circ} \mathrm{C}$ ). In contrast, defects, which are created through ion irradiation, react readily with water, leading to a partial removal of the damage layer. The dominant oxidic group at the surface is the carbonyl group, although the presence of other oxygen containing functional groups can not be excluded. The reduction in damage through the interaction with water is accompanied by an increase in the carbonyl concentration, which indicates that the dissociation of water at the diamond surface might follow a different pathway than on silicon surfaces. In the irradiation of the damaged surface with oxygen ions the chemical etching dominates and the overall damage is reduced. This study also shows that the surface defects can be decisive for the long term stability of diamond surface properties.

\section{Acknowledgements}

The authors wish to thank W. Haenni, and D. Morel from the Centre Suisse d'Electronique et Microtechnique, Neuchâtel, Switzerland, for providing the diamond substrates, which were indispensable for the experiments. We also gratefully acknowledge the financial support of the present work by the Swiss Priority Programm on Materials Research carried out under the auspices of the trinational , D-A-CH" cooperation involving Germany, Austria and Switzerland on the „Synthesis of Superhard Materials“. 


\section{References}

1 A. Perret, W. Haenni, N. Skinner, X.-M. Tang, D. Gandini, C. Cominellis, B. Correa, and G. Foti, Diamond and Rel. Mat. 8, 820 (1999).

2 J. B. Cui, J. Ristein, and L. Ley, Phys. Rev. B 59, 5847 (1999).

3 J. S. Foord, K. P. Loh, and R. B. Jackman, Surf. Sci. 399, 1 (1998).

4 S. Matsumoto and N. Setaka, Carbon 17, 485 (1979).

5 T. E. Beerling and C. R. Helms, Appl. Phys. Lett. 64, 288 (1994).

6 L. M. Struck and M. P. D'Evelyn, J. Vac. Sci. Technol. A 11, 1992 (1993).

7 J. E. Field, The Properties of Natural and Synthetic Diamond (Academic Press, London, 1992).

8 S. Matsumoto, H. Kanda, Y. Sato, and N. Setaka, Carbon 15, 299 (1977).

9 S. Evans and J. M. Thomas, Proc. Ro:Soc.London A353, 103 (1977).

10 L. J. Huang, I. Bello, W. M. Lau, S. T. Lee, P. A. Stevens, and B. D. DeVries, J. Appl. Phys. 76, 7483 (1994).

11 P. Reinke, G. Francz, P. Oelhafen, and J. Ullmann, Phys. Rev. B 54, 7067 (1996).

12 A. Knop-Gericke, M. Hävecker, T. Neisius, and T. Schedel-Niedrig, Nuclear Instruments and Methods in Physics Research A 406, 311(1998).

13 E. Diez, W. Braun, A. M. Bradshaw, and R. L. Johnson, Nucl. Instrum. Meth. A 239, 359 (1985).

14 J. Stöhr, NEXAFS Spectroscopy, Vol. 25 (Springer, Berlin Heidelberg, 1992).

15 T. Schedel-Niedrig, D. Herein, H. Werner, M. Wohlers, R. Schlögl, G. Francz, P. Kania, P. Oelhafen, and C. Wild, Europhys. Lett. 31 , 461 (1995).

16 G. Comelli, J. Stöhr, C. J. Robinson, and W. Jark, Phys. Rev. B 38, 7511 (1988).

17 F. L. Coffman, R. Cao, P. A. Pianetta, S. Kapoor, M. Kelly, and L. J. Terminello, Appl. Phys. Lett. 69, 568 (1996).

18 G. R. Wight and C. E. Brion, J. Electron. Spectosc. and Rel. Phen. 4, 25 (1974).

19 D. A. Outka, J. Stöhr, R. J. Madix, H. H. Rotermund, B. Hermsmeier, and J. Solomon, Surf. Sci. 185, 53 (1987).

20 E. Schröder-Bergen and W. Ranke, Surf. Sci. 236, 103 (1990).

21 Y. J. Chabal and S. B. Christman, Phys. Rev. B 29, 6974 (1984). 